\title{
WEAK STRUCTURAL SYNTHESIS FOR CERTAIN BANACH ALGEBRAS $\left({ }^{1}\right)$
}

\author{
BY \\ PAUL CIVIN
}

1. Introduction. In the study of the group algebra, $L^{1}(G)$, of a locally compact abelian group $G$, considerable attention has been given (e.g. $[1 ; 4]$ ) to the question of approximation in some sense of elements in the conjugate space, $L^{\infty}(G)$, by linear combinations of characters of $G$, taken from the spectrum or a neighborhood of the spectrum of a given member of $L^{\infty}(G)$. Similar questions were considered [3] relative to other commutative algebras associated with a locally compact abelian group. Such statements are frequently given in dual form as statements concerning certain ideals in the algebra.

The object of the present work is to exhibit that similar types of approximations are possible with respect to elements in the conjugate space of certain Banach algebras, whether or not these algebras are commutative, and without any association of the algebra with a group.

Throughout this work we restrict our attention to Banach algebras in which each proper closed 2 -sided ideal is contained in a maximal modular left ideal. In addition to the aforementioned algebras, $L^{1}(G)$, the class contains various $B^{*}$-algebras $[5$, p. 232], and various completely regular algebras (GS algebras) [9, p. 188].

In $\S 3$, we associate certain subsets of the structure space of $B$ to individual elements of $B^{*}$, and obtain some of the properties induced by this association. In $\$ 4$, we obtain two forms of a weak structural synthesis, one of which involves no further assumptions on the algebra than already indicated.

2. Notation. Throughout the sequel, $B$ will denote a Banach algebra with the property that any proper closed 2-sided ideal is contained in a maximal modular left ideal. The operator defined on $B$ by left (right) multiplication by $x \in B$ will be denoted by $L_{x}\left(R_{x}\right)$. For a subset $A$ of $B$, the quotient $A: B$ is defined by $A: B=\{x \in B \mid x y \in A, \forall y \in B\}$.

As usual, the conjugate space of $B$ will be denoted by $B^{*}$. For a subspace $V$ of $B^{*}$, we let $V^{\top}=\{x \in B \mid f(x)=0, \forall f \in V\}$, while for a subspace $A$ of $B$ we let $A^{\perp}=\left\{f \in B^{*} \mid f(x)=0, \forall x \in A\right\}$. We say that a subspace of $B^{*}$ is left (right) invariant, if the subspace is invariant under each of the operators $L_{x}^{*}\left(R_{x}^{*}\right), x \in B$. For $\phi \in B^{*}$, we let $V_{\phi}$ denote the smallest $w^{*}$-closed invariant subspace containing $\phi$.

The letters $h$ and $k$ are reserved as symbols for the hull and kernel, each

Received by the editors March 15, 1961 and, in revised form, July 28, 1961.

(1) This research was supported by the National Science Foundation, grant NSF-G-14111. 
taken with reference to the structure space II, i.e. the collection of primitive ideals of $B$ taken with the hull-kernel topology (see [8, p. 78]).

We say that an algebra $B$ has an approximate right identity if there exists a net $\left\{y_{\alpha}\right\}$ in $B$ such that for any $x \in B, x=\lim x y_{\alpha}$.

3. Structure and conjugate spaces. We start by relating various notions concerning the algebra $B$ to properties of the conjugate space.

3.1. Lemma. If $V$ is a $w^{*}$-closed subspace of $B^{*}$, then $V$ is left (right) invariant if and only if $V^{\top}$ is a left (right) ideal of $B$.

Proof. Let $f \in V, y \in V^{\top}$ and $z \in B$. The equality $L_{z}^{*} f(y)=f\left(L_{z} y\right)=f(z y)$ yields the equivalence statement for left ideals, with a corresponding relation yielding the statement for right ideals.

3.2. Lemma. Let $A$ be a left ideal in $B$. The quotient $A: B$ is given by the equation $A: B=\left\{x \in B \mid L_{x}^{*} f=0, \forall f \in A^{\perp}\right\}$.

Proof. Suppose $L_{x}^{*} f=0, \forall f \in A$. For all $y \in B, 0=L_{x}^{*} f(y)=f(x y)$. Consequently $x y \in A, \forall y \in B$, and $x \in A: B$. The opposite set inequality is seen in the same manner.

As the duals of standard ring theoretic properties, we have the following statements.

3.3. Lemma. (i) Let $A$ be a modular left ideal in $B$. The smallest $w^{*}$-closed invariant subspace of $B^{*}$ which contains $A^{\perp}$ is $(A: B)^{\perp}$.

(ii) Let $V$ be a $w^{*}$-closed invariant subspace of $B^{*}, V \neq(0)$. Then there is a primitive ideal $Q$ in $B$ such that $Q^{\perp} \subset V$.

3.4. Lemma. Let $V$ be a $w^{*}$-closed invariant subspace of $B^{*}$, then $h V^{\top}$ $=h\left(V^{\top}: B\right)$.

Proof. Let $P \in h V^{\top}$. Say $P=M: B$ where $M$ is a maximal modular left ideal of $B$. Then $V^{\top} \subset P \subset M$, so $\left(V^{\top}: B\right) \subset(M: B)=P$, and $P \in h\left(V^{\top}: B\right)$.

Since $V^{\top}$ is an ideal of $B, V^{\top} \subset\left(V^{\top}: B\right)$ and consequently $h\left(V^{\top}: B\right) \subset h V^{\top}$. Thus $h V^{\top}=\left(V^{\top}: B\right)$.

A question which is suggested by Lemma 3.4 is whether $I: B$ is the largest 2 -sided ideal with the same hull as the 2 -sided ideal $I$. The answer can be seen to be negative. Take for $B$ the algebra $L^{* *}(G)$, the algebra of the second conjugate space of the group algebra of a locally compact infinite abelian group (see [2]). If $I=\{0\}$, then $I: B=I$ since $B$ has a right identity. Thus $h(I)=h(I: B)=\Pi$. However, the largest 2 -sided ideal with hull equal to $\Pi$ is clearly the radical of $B$, and the latter is different from $I$.

In the sequel, if $\phi \in B^{*}$, we write $h(\phi)$ for $h\left(V_{\phi}^{\top}\right)$.

3.5. LEMma. (i) If $x \in B$ and $\phi \in B^{*}$, then $h\left(L_{x}^{*} \phi\right) \subset h(\phi)$.

(ii) If $\phi, \psi \in B^{*}$, then $h(\phi+\psi) \subset h(\phi) \cup h(\psi)$.

(iii) If $\phi, \psi \in B^{*}$ and if $h(\phi) \cap h(\psi)=\varnothing$, then $h(\phi+\psi)=h(\phi) \cup h(\psi)$. 
Proof. The first statement is immediate. Let $x \in\left(V_{\phi}^{\top}: B\right) \cap\left(V_{\psi}^{\top}: B\right)$. Then $L_{x}^{*} f=0$ for $f \in V_{\phi} \cup V_{\psi}$, and thus $L_{x}^{*}(\phi+\psi)=0$. If $T$ denotes any product of adjoints of operators of the type $L_{y}$ or $R_{z}$, then $L_{x}^{*} T(\phi+\psi)=0$. Consequently, by the $w^{*}$-continuity of the operators $L_{x}^{*}$, we obtain $L_{x}^{*} f=0$ for all $f \in V_{\phi+\psi}$. It follows from Lemma 3.2 that $\left(V_{\phi}^{\top}: B\right)\left(V_{\psi}^{\top}: B\right) \subset\left(V_{\phi}^{\top}: B\right) \cap\left(V_{\psi}^{\top}: B\right) \subset\left(V_{\phi+\psi}^{\top}: B\right)$. If $P \in h(\phi+\psi)=h\left(V_{\phi+\psi}^{\top}\right)$, then $P \supset\left(V_{\phi}^{\top}: B\right)\left(V_{\psi}^{\top}: B\right)$. It now follows from Theorem 2.29 of [8] that either $P \supset\left(V_{\phi}^{\top}: B\right)$ or that $P \supset\left(V_{\psi}^{\top}: B\right)$. Another application of Lemma 3.4 yields $P \in h(\phi) \cup h(\psi)$.

Suppose next that $h(\phi) \cap h(\psi)=\varnothing$. Let $Q \in h(\phi) \cup h(\psi)$. Suppose $Q \in h(\phi)$, so by hypothesis $Q \notin h(\psi)$. By a minor modification of a formula noted above, $\left(V_{\phi+\psi}^{\top}: B\right)\left(V_{\psi}^{\top}: B\right) \subset\left(V_{\phi+\psi}^{\top}: B\right) \cap\left(V_{\psi}^{\top}: B\right) \subset\left(V_{\phi}^{\top}: B\right)$. It then follows by Lemma 3.4 that $Q \supset\left(V_{\phi+\psi}^{\top}: B\right)\left(V_{\psi}^{\top}: B\right)$. Consequently, either $Q \supset\left(V_{\phi+\psi}^{\top}: B\right)$ or $Q \supset\left(V_{\psi}^{\top}: B\right)$. Our supposition that $Q \notin h(\psi)$ together with Lemma 3.4 yields $Q \in h(\phi+\psi)$ as desired.

\section{Weak structural synthesis.}

4.1. Lemma. Let $\theta$ be a subset of $\Pi$. The smallest $w^{*}$-closed invariant subspace of $B^{*}$ which contains all $P^{\perp}$ for $P \in \theta$ is $k(\theta)$.

Proof. Let $S$ denote the subspace described in the lemma. Since $P^{\perp} \subset S$ for all $P \in \theta$, it follows that $S^{\top} \subset k(\theta)$. Suppose that $f \in S$ and $f \notin k(\theta)^{\perp}$. Then there is an $x \in k(\theta)$ such that $f(x)=1$. It follows that there exist $P_{\alpha, i} \in \theta$, $f_{\alpha, i} \in P_{\alpha, i}^{\perp}$ and $f=w^{*}-\lim \sum_{i=1}^{n(\alpha)} f_{\alpha, i}$. But then $f(x)=\lim \sum_{i=1}^{n(\alpha)} f_{\alpha, i}(x)=0$, which is a contradiction.

4.2. Lemma. Let $B$ be a semi-simple Banach algebra, each of whose proper closed 2-sided ideals is contained in a maximal modular left ideal. Let I be a closed 2-sided ideal in $B$ and let $\theta$ be an open subset of $\Pi$ such that $h(I) \subset \theta$. Then $k(\theta) \subset(I: B)$. Conversely, suppose that $K$ is a closed set in $\Pi$. If for each open set $U$ such that $U \supset K$, we have $k(U) \subset(I: B)$, then $h(I) \subset \cap \bar{U}$, where the intersection is taken over all the open sets $U$ which contain $K$. In particular, if $\Pi$ is Hausdorff, then $h(I) \subset K$.

Proof. Let $x \in k(\theta)$. Let $\psi=L_{x} \phi$, for $\phi \in I^{\perp}$. Then $V_{\psi} \subset I^{\perp}$ and $I \subset V_{\psi}^{\top}$. If $V_{\psi} \neq(0)$, there is a $Q \in \Pi$, such that $Q \in h(\psi)$, whence $Q \in h(I) \subset \theta$. Let $\theta^{\prime}$ denote the complement of $\theta$ in $\Pi$. Since $\theta$ is open, $\theta^{\prime}=h k\left(\theta^{\prime}\right)$ and therefore $Q \nsupseteq k\left(\theta^{\prime}\right)$. Let $y \in k\left(\theta^{\prime}\right), y \notin Q$. It follows from the semi-simplicity of $B$ that $x y=0$. Consequently, $L_{v}^{*} \psi=L_{v}^{*} L_{x}^{*} \phi=0$. The same result holds if $\psi$ is replaced by any of $L_{z}^{*} \psi, L_{z}^{*} R_{w}^{*} \psi$, or $R_{z}^{*} \psi$. Thus it follows that $L_{y}^{*} f=0$, for any $f \in V_{\psi}$. Lemma 3.2 then yields $y \in\left(V_{\psi}^{\top}: B\right)$. Now $Q \in h(\psi)=h\left(V_{\psi}^{\top}: B\right)$, so $y \in Q$. This contradicts an earlier observation, and consequently $V_{\psi}=(0)$, so $\psi=0$. Since $\phi$ was arbitrary in $I^{\perp}$, it follows from Lemma 3.2 that $x \in(I: B)$.

Let $K$ be a closed set in $\Pi$. Suppose that $(I: B) \supset k(\theta)$ for each open set $\theta$ such that $\theta \supset K$. Thus $h k(\theta) \supset h(I: B)=h I$, and $h I \subset \cap h k(\theta)$, where the intersection is over all the open sets $\theta$ such that $\theta \supset K$. If $\Pi$ is Hausdorff, it follows 
from the local compactness of the structure space $[8$, p. 79] that the indicated intersection is $K$. This completes the proof of Lemma 4.2.

Let $I$ be a 2 -sided ideal in $B$. Let $J(I)$ be the smallest 2 -sided ideal in $B$ which contains each $k(\theta)$, where $\theta$ is open in $\Pi$ and $\theta \supset h(I)$. It is easily seen that $J(I)=\{x \in B \mid x \in k(\theta)\}$ for some open set $\theta$ in $\Pi$ with $\theta \supset h(I)$.

4.3. TheOREM. Let $B$ be a semi-simple Banach algebra each of whose proper closed 2-sided ideals is contained in a maximal modular left ideal. If I is a closed 2-sided ideal in $B$, then $J(I) \subset(I: B)$. If either $B$ has an approximate right identity, or $I$ is a modular ideal then $J(I) \subset I$.

Proof. In view of the equivalent form of the definition of $J(I)$, the first part of the statement follows immediately from the first part of Lemma 4.2. Under each of the conditions of the second assertion of the theorem $I: B=I$, and the proof is complete.

It should be noted that the conclusion $J(I) \subset I$ is not always available. An example is given by $\mathrm{H}$. Mirkil [7] of a commutative Banach algebra, $A$, which is Tauberian and whose space of maximal modular ideals (i.e., structure space) is discrete. Mirkil shows that there is an $x \in A$ such that $x \notin(x A)^{-}=I$. If $\mu \in h(I)$, then $\mu(x y)=0$ for all $y$ in $A$ and thus $\mu(x)=0$. Thus $x \in k h(I)$ $\subset J(I)$, since $\Pi$ is discrete. If $J(I) \subset I$, it then follows that $I=k h(I)$, and therefore that $x \in I$, which is a contradiction.

A dual form of Theorem 4.3 may be stated in terms of elements of $B^{*}$. Let $\phi \in B^{*}$, then the initial conclusion of the theorem would read $J\left(V_{\phi}^{\top}\right) \subset\left(V_{\phi}^{\top}: B\right)$. If we impose further hypotheses, we may obtain the more familiar conclusion $J\left(V_{\phi}^{\top}\right) \subset V_{\phi}^{\top}$. We say that the algebra $B$ satisfies the condition $\left(^{*}\right)$ if each point $P \in \Pi$ is contained in an open set whose closure has a modular kernel. The condition $\left({ }^{*}\right)$, when applied to the space of maximal modular 2 -sided ideals, is one of the two defining conditions of the completely regular algebras [8] (i.e., the $G S$ algebras of Willcox [9]).

4.4. Lemma. Let $B$ satisfy condition $(*)$. Suppose, for $\phi \in B^{*}$, that $V_{\phi} \neq B^{*}$ and $h(\phi)$ is compact. Then there exists an open set $\theta$ in $\Pi$ such that $\theta \supset h(\phi)$ and $k 6$ is a modular 2-sided ideal.

Proof. The result is immediate from condition $(*)$ and a standard compactness argument.

4.5. Lemma. Let B be semi-simple and let I be a closed 2-sided ideal in B. Let $\theta$ be open in $\Pi$ with $h I \subset \theta$ and $k \theta$ a modular 2-sided ideal. Then $I$ is a modular 2-sided ideal.

Proof. Let $\theta^{\prime}$ be the complement of $\theta$. By semi-simplicity $k \theta \cap k \theta^{\prime}=(0)$. Let $j$ be a relative identity modulo $k \theta$. Then for $x \in k \theta^{\prime}, j x-x \in k \theta \cap k \theta^{\prime}$, so $j x=x$. We show next that the ideal $I+k \theta^{\prime}$ is dense in $B$. Suppose that the closure of $I+k \theta^{\prime}$ is proper. The basic hypothesis on $B$ then implies that there 
exists a $P \in \Pi$ such that $P \supset I+k \theta^{\prime}$. Thus $P \in h I \subset \theta$, while $P \in h k \theta^{\prime}=\theta^{\prime}$, which is a contradiction. Let $x \in B$. There exist $x_{n} \in I, y_{n} \in k \theta^{\prime}$ such that $x=\lim \left(x_{n}+y_{n}\right)$. Then $j x-x=\lim \left\{j\left(x_{n}+y_{n}\right)-\left(x_{n}+y_{n}\right)\right\}=\lim \left(j x_{n}-x_{n}\right)$. Since $I$ is closed and $x_{n} \in I, j x-x \in I$. Likewise $x j-x \in I$.

4.6. Corollary. Let $B$ be a semi-simple Banach algebra which satisfies $\left({ }^{*}\right)$ and each of whose proper closed 2-sided ideals is contained in a maximal modular left ideal. Let $\phi \in B$ with $h(\phi)$ compact. Then $J\left(V_{\phi}^{\top}\right) \subset V_{\phi}^{\top}$.

Proof. If $V_{\phi}^{\top} \neq B^{*}$, the result follows immediately from Theorem 4.3 and Lemmas 4.4 and 4.5. If $V_{\phi}^{\top}=B^{*}$, the conclusion is automatic.

It should be noted that for certain algebras $B$ it may happen that $J(I)$ $=(0)$ for each 2-sided ideal 1, e.g., in any simple semi-simple Banach algebra.

\section{REFERENCES} 238.

1. Arne Beurling, On the spectral synthesis of bounded functions, Acta Math. 81 (1948), 225-

2. Paul Civin and Betram Yood, The second conjugate space of a Banach algebra as an algebra, Pacific J. Math. 11 (1961), 847-870.

3. Yngve Domar, Harmonic analysis based on certain commutative Banach algebras, Acta Math. 96 (1956), 1-56.

4. Roger Godement, Théorèmes taubériens et théorie spectrale, Ann. Sci. Ecole Norm Sup. (3) 64 (1947-48), 119-138.

5. Irving Kaplansky, The structure of certain operator algebras, Trans. Amer. Math. Soc. 70 (1951), 219-255. 1953.

6. L. H. Loomis, An introduction to abstract harmonic analysis, Van Nostrand, New York,

7. H. Mirkil, A counterexample to discrete spectral synthesis, Compositio Math. 14 (1960), 269-273.

8. C. E. Rickart, General theory of Banach algebras, Van Nostrand, New York, 1960.

9. A. B. Willcox, Some structure theorems for a class of Banach algebras, Pacific J. Math. 6 (1956), 177-192.

UNIVERSITY OF FLORIDA,

GAINESVILLE, FloRIDA

UNIVERSITY OF OREGON,

EUGENE, Oregon 